\title{
VIOLENCIA INTRAFAMILIAR EN TIEMPO DE PANDEMIA EN UNA COMUNA DEL DISTRITO ESPECIAL TURÍSTICO Y CULTURAL EN EL DEPARTAMENTO DE LA GUAJIRA
}

\section{INTRAFAMILY VIOLENCE IN TIMES OF PANDEMIC IN A COMMUNITY OF THE SPECIAL TOURIST AND CULTURAL DISTRICT IN THE DEPARTMENT OF LA GUAJIRA}

\section{Clara Judith Brito Carrillo' \\ Juannys Chiquillo Rodelo \\ Yenifeth Omaira Blanco Torres ${ }^{3}$}

Universidad de La Guajira

1 Mcs. Desarrollo y Gestión de empresas sociales Gerencia Social. Trabajadora Social, Docente e investigadora Universidad de La Guajira. Colombia. Autor y coautor de artículos de revistas científicas e indexadas, capítulo de libros, libros a nivel nacional e internacional. Investigador asociado, líder Grupo de Investigación: Encuentro con la investigación. Email.clarabrito@ uniguajira.edu.co . https://orcid.org/0000-0001-8788-7326

2 Doctora en Ciencias Política, Magíster en Desarrollo Familiar. Psicóloga, Docente e investigadora de la Universidad de La Guajira Colombia. Autor y coautor de artículos de revistas científicas e indexadas, capítulo de libros, libros a nivel nacional e internacional. Grupo de Investigación: Tamaskal jchiquillo@uniguajira.edu.co. https://orcid.org/0000-0001-8673-4838

3 Mcs. En desarrollo integral de niños niñas y adolescentes. Especialista en Gerencia y Salud. Trabajadora Social, Docente Universidad de la guajira. Autor y coautor de artículos de revistas científicas e indexadas, capítulo de libros, libros a nivel nacional e internacional. Grupo de Investigación: Palabra y Sociedad. Email. yoblancot@uniguajira.edu.co. https://orcid. org/0000-0002-5530-8526 


\section{RESUMEN}

El presente artículo analiza el aumento de la violencia intrafamiliar en tiempos de pandemia en el departamento de La Guajira, municipio de Riohacha. Esta investigación es de corte cuantitativo, descriptivo, correlacional de campo, la población se caracterizó por una muestra de Veinte familias, lo cual permitió realizar un análisis minucioso sobre la multiplicidad de factores de riesgo y vulnerabilidad que se suscita por la violencia intrafamiliar física y psicológica, así mismo verbal y sexual. Los resultados muestran en los victimarios inconvenientes de tipo psicológico y social, carencias afectivas, inadecuadas relaciones parento- filiares y de convivencia familiar, ausencia de acciones socio psicoeducación en tiempo de confinamiento sobre el fenómeno presentado, y una influencia marcada cultural del papel de la familia en la sociedad. A manera de conclusión, la situación de contingencia y sus diferentes manifestaciones de violencia intrafamiliar requiere medidas por parte de los diferentes entes gubernamentales, además de la implementación de medidas de salud que propendan por mitigar los efectos devastadores sobre las personas en tiempos de confinamiento obligatorio. Aunque las medidas de protección limiten la intervención o haga que sea más restringido desde el área de la salud mental se pueden implementar herramientas desde las instituciones para abordar los efectos nocivos de la violencia intrafamiliar en el departamento de La Guajira, municipio de Riohacha.

\section{PALABRAS CLAVE:}

Familia, violencia intrafamiliar, tiempo de pandemia

\section{ABSTRACT}

This article makes visible through the general objective to analyze the increase in intrafamily violence in times of pandemic in the department of the Guajira, municipality of
Riohacha, in this order of ideas, from different methodological perspectives, this research is quantitative, descriptive, correlational field, the population was characterized by a sample of ten families, which allowed a detailed analysis of the multiplicity of risk and vulnerability factors that arises from physical and psychological intrafamily violence, also verbal and sexual, as well as likewise, the results show psychological and social inconveniences in the victimizers, lack of affection, inadequate parental relations and poor family coexistence, absence of sociopsychoeducation actions in time of confinement on the phenomenon presented, and a marked cultural influence of the role of the family in society. In conclusion. The contingency situation and its different manifestations of domestic violence require measures by the different government entities, in addition to the implementation of health measures that tend to mitigate the devastating effects on people by mandatory confinement, although the protection measures limit the intervention or make it more restricted from the mental health area, tools can be implemented from the institutions to address the harmful effects of intrafamily violence in times of pandemic in the department of the Guajira, municipality of Riohacha

\section{KEYWORDS:}

Family, domestic violence, time of pandemic

\section{INTRODUCCIÓN}

La violencia intrafamiliar es un tema de estudio importante en las ciencias sociales, a medida que ha aumentado el fenómeno, también se ha hecho consciente su impacto en diversas áreas de la vida social en el campo de la salud, ya que se atenta contra la integridad física, mental y social de la persona violentada

En este orden de ideas, La violencia intrafamiliar, se convierte en un problema complejo que afecta considerablemente generando inestabilidad, dada sus diferentes 
manifestaciones se requiere de espacios de análisis y reflexión de manera profundad que permitan la implementación de programas de intervención y prevención psicosocial y por ende de equipos interdisciplinarios, que conduzcan al fortalecimiento de acciones direccionadas por los entes gubernamentales, quienes ejercen el control para detener los riesgos psicosociales implícitos en esta problemática social, cuyo aumento es evidente por el confinamiento producido por el Covid-19

Gracias a los avances científicos y a las prácticas clínicas que se ocupan de la salud mental y los abordajes psicosociales en general, se puede favorecer el amplio panorama respecto a tratamientos adecuados y de elección para corregir cada problema mental, mitigar y prevenir los inconvenientes que están surgiendo de tipo psicosocial. Desde un abordaje psicológico se busca fortalecer factores de protección tanto internos como externos, que protejan al individuo de la posibilidad de desarrollar cuadros psicopatológicos posteriores. Los factores de orientación son de apoyo y protección familiar, apoyo social próximo, (amigos y grupos filiales), apoyo social general, social, institucional y psicológico.

Los resultados de la siguiente investigación, visibiliza que ante esta crisis global del Covid_19, se han suscitado situaciones que inestabilizan la dinámica familiar, por la misma convivencia sociocultural cuyas repercusiones psicológicas son de gran alcance dadas sus implicaciones, por su parte, dada las características y ubicación geoestratégicas del departamento de La Guajira, surge la necesidad de investigar sobre las políticas públicas en los programas sociales que abordan situaciones conflictivas y que los cual requiere de unos procesos de promoción y sensibilización dirigidos al mejoramiento de la convivencia familiar, para reducir la agresión a la mujer y los niños y niñas a través de la violencia intrafamiliar. No ajeno a ello, al analizar las diferentes manifestaciones de la violencia doméstica, o intrafamiliar, la cual posee afectaciones en la descomposición de la dinámica familiar, conlleva a que se revisen causa y consecuencias a través de los resultados de la investigación titulada: Aumento de las diferentes manifestaciones de violencia intrafamiliar en tiempo de pandemia en el departamento de La Guajira.

En este sentido, La Violencia Intrafamiliar es una de las expresiones de agresión y maltrato que más afecta la formación y el desarrollo integral de la personalidad de un individuo sea niño, niña, adolescente o adulto (Pavón y. Santamaría, 2010).

No obstante, las problemáticas que se han generado en estos momentos productos del aislamiento social del Covid-19, se convierten en una fuente de análisis y reflexión frente al fenómeno del aumento indiscriminada de la violencia intrafamiliar. Así mismo, se destacan las consideraciones de la consideran que la violencia familiar, incluye a todas las formas de abuso en las relaciones entre los miembros de una familia. Se refieren a las distintas formas de relación abusiva que caracterizan de modo permanente o cíclico al vínculo familiar. Las relaciones de abuso son aquellos vínculos caracterizados por el ejercicio de la violencia de una persona hacia otra. Enfatizan que cualquier miembro de la familia, independientemente de su raza, sexo y edad, puede ser agente o víctima de las relaciones abusivas. (Corsi y Bobino, 2014)

Dentro de este contexto, se puede afirmar que las situaciones conflictivas que se enuncian a través de los medios de comunicación en estos momentos merecen atención por parte de los entes gubernamentales y no gubernamental, dadas sus implicaciones para la vida integral de la familia debido a que la violencia intrafamiliar inestabilidad la condición humana de la mujer y por ende del núcleo familiar. 
Según el reporte de medicina Legal, publicado por Alvarado (Septiembre 12, 2018) se destaca

En Cada día se reportan 136 casos de violencia de pareja en Colombia. El 86 por ciento de las víctimas fueron mujeres, según cifras preliminares de Medicina Legal. En estos se han visto afectados 24.830 mujeres (86\%) y 4.026 hombres. Del total de víctimas, 762 fueron menores de edad. Por edades, los más afectados tienen entre 20 a 24 años (5.928). Siguieron personas entre 25 y 29 (6.313) y de 30 a 34 años (5.134). Hasta el momento, el mes que más casos registro fue marzo, con 4.454. Siguieron mayo (4.244), abril (4.224) y febrero (4.139).

Cabe resaltar las afirmaciones realizadas por Prins (Octubre 31, 2017) cuando expresa "sigue en aumento la violencia de género en el departamento: 844 casos en lo que va del 2017"

"La violencia contra la mujer sigue en aumento en el departamento de La Guajira. Así lo revelan las cifras entregadas por el Sistema Nacional de Vigilancia en Salud Pública -Sivigila-, el cual reporta 844 casos en lo que va corrido del 2017. De acuerdo al informe de la Secretaría de Salud departamental, intervenida por el Gobierno nacional a través de una Asunción Temporal, para esa misma fecha en el 2016 se registraron 663 casos, presentándose un aumento de 181 sucesos. Los guarismos indican que en el caso del Distrito de Riohacha se han presentado 393 hechos de violencia contra la mujer; seguido de Villanueva, donde se reportan 142 episodios.
En el listado siguen Maicao, 65; Dibulla, 59; y San Juan del Cesar, 69. En Fonseca, los sucesos alcanzan a 35; Uribia, 24; Barrancas, 14; Manaure, 11 casos; Hatonuevo, 10; El Molino, 7; Urumita, 6; Albania, 4; La Jagua del Pilar, 3; y Distracción, 2.

En el citado informe quedó claro que las mujeres solteras son las principales víctimas, además, que la mayoría de las agresiones se presentan los fines de semana, y que el 95 por ciento de los casos pertenecen a grupos indígenas.

Asimismo, se indican que las principales agresiones a las mujeres ocurren en el hogar, y que los responsables son sus actuales parejas o antiguos compañeros sentimentales. Las cifras han llamado la atención de la comunidad, quienes están solicitando a las autoridades que manejan el tema, prestarle un poco más de atención para concertar algunas acciones y tratar de bajar esos índices.

Por su parte, La violencia intrafamiliar abarca todo aquel comportamiento de violencia física, sexual o psicológica que llega a poner en situación de peligro la seguridad o el bienestar de una persona del grupo familiar; el comportamiento de las personas que recurren tanto a la fuerza física como al chantaje emocional; las amenazas que dan lugar al recurso a la fuerza física, entre las que se incluye la violencia sexual, tanto en la familia como en el hogar. (Ruiz, 2016)

Es por ello, que el grupo vulnerable ante la violencia familiar lo constituyen, sin duda, las niñas, niños y adolescentes. Ya que, además de no asistir a las escuelas, han tenido educación a distancia, debiendo adaptarse rápidamente a esta forma de aprender y a comprender conceptos de manera remota. Aunado a lo anterior, otros miembros de la familia también están todo el día en casa, por lo que deben compartir materiales, aparatos tecnológicos 
de trabajo y un espacio que no siempre es suficiente. (Gómez y Sánchez, 2020. p.p 14.15)

\section{DESARROLLO}

A Nivel nacional, se puede visibilizar las apreciaciones del documento, orientaciones Para La Salida de Niñas, Niños y Adolescentes a Espacio Público durante la Pandemia Por Covid-19 En Colombia, hace referencia a la temática investigada en la cual se puede verificar...

Situaciones especiales de salud: Existe un riesgo de aumento de violencia física, psicológica $y$ sexual durante el Aislamiento Preventivo Obligatorio que se puede registrar en el hogar, por estar conviviendo con su agresor; los cuales no se identifican fácilmente desde los servicios de salud, educación o la comunidad. Lamentablemente relacionarse con eventos de embarazos no deseados por abuso sexual, enfermedades de trasmisión sexual o afectaciones físicas que pueden llevar a discapacidad; así como afectaciones de la salud mental. Divulgar de manera masiva el alcance de la medida y especialmente los grupos de niños, niñas y adolescentes en las cuales no aplica esta medida; así como los canales $y$ estrategias para favorecer el cuidado en casa hasta tanto se encuentren las condiciones que permitan la salida de estos grupos. Esto incluye fortalecer todas las acciones para promover la actividad física, la alimentación saludable, el cuidado de la salud mental, la prevención de las violencias, el cuidado a los cuidadores, entre otros, al interior de todas las familias. (Ministerio de Salud y Protección Social, (MinSalud, 2020 p.p 5-8).

La temática de la violencia intrafamiliar, posee sus connotaciones para la Organización Mundial de la salud [OMS, 2012], ha propuesto un conjunto de determinantes estructurales que generan estratificación social e inequidades en salud, entre los cuales incluye indicadores tradicionales, como el ingreso y la educación, y reconoce explícitamente a la etnia, el género y la sexualidad, lo que constituye un verdadero avance en contraposición con los enfoques biologicistas tradicionales. Como determinantes intermediarias se consideran aquellas que establecen diferencias en la exposición y vulnerabilidad e incluyen: condiciones de vida, disponibilidad de alimentos, conductas poblacionales

El aumento de la violencia interpersonal en tiempos de crisis es un hecho bien documentado. Pero, el insuficiente número de denuncias, dificulta la respuesta y la recopilación de datos, ya que menos del $40 \%$ de las mujeres que sufren violencia buscan ayuda o denuncian el delito. De las mujeres que sí deciden solicitar ayuda, menos del $10 \%$ se dirigen a la policía. (Departamento de Comunicación Global de las Naciones Unidas, (DCGNU, 2020)

Las secuelas psicológicas son difíciles de identificar ya que no dejan marcas físicas, no obstante, se produce abuso cognitivo y emocional. Existen diversas teorías que intentan explicar el fenómeno producido por la violencia intrafamiliar, una de ellas es la que explica el ciclo de violencia en el que el victimario va desgastando la autoestima de su víctima en un proceso en el que la hace adaptar al maltrato, demostrando su poder y provocando intimidación e indefensión aprendida, que logra que la víctima permanezca sumisa como forma 
de prevenir el castigo por parte de su victimario. (Hernández et al.,2014. p. 34).

En consideración, se resalta, Palacios y Rodrigo (como se citó en Gallego (2011) afirman que la familia se concibe como una asociación de personas que comparten propósitos de vida y que desean mantenerse unidos en el tiempo. Según Torres et al. (2008, como se citó en Gallego, 2011), la familia es "un sistema de interrelación biopsicosocial que media entre el individuo y la sociedad y se encuentra integrada por un número variable de individuos, unidos por vínculos de consanguinidad, unión, matrimonio o adopción" (p. 24).

La violencia es definida como el uso de poder en forma de intimidación contra: uno mismo, una comunidad o una persona, ocasiona posibles lesiones a nivel físico, psicológico, Tx de desarrollo o la pérdida de la vida. Asimismo, la OMS en su informe anual categoriza la violencia en 3 divisiones, violencia dirigida contra uno mismo, violencia interpersonal y violencia colectiva. Organización Mundial de la salud (OMS, 2012).

\section{* VIOLENCIA INTRAFAMILIAR}

Las víctimas de violencia sexual y familiar, comparten características que indican vulnerabilidad, lo que sugiere que la atención para ambos grupos podría combinarse mejor en un solo centro de asalto. De esta manera, las víctimas pueden hacer uso de los mismos servicios y conocimientos de violencia de género. Uno de los objetivos principales de los centros de asalto es proporcionar atención psicosocial de seguimiento y facilidades para reportar (Flores y González, 2015).

"La violencia física es la acción o conducta, que causa daño a la integridad corporal o a la salud. Se incluye el maltrato por negligencia, descuido o por privación de las necesidades básicas, que hayan ocasionado daño físico o que puedan llegar a ocasionarlo, sin importar el tiempo que se requiera para su recuperación" Comisión Económica para América Latina y el Caribe (CEPAL, 2016)

En este sentido,

El autor, Walker (2003) explica que, para comprender la violencia intrafamiliar en las relaciones de pareja, se deben tener en cuenta tres fases: La primera fase es la de acumulación de tensión, que se entiende por el hecho de que la víctima percibe que el agresor se vuelve cada vez más irritable, respondiendo con hostilidad y encontrando motivos de conflicto en cada situación. Aparecen agresiones psicológicas, golpes o empujones, amenazas, etc. La mujer, temerosa, hace todo lo posible para no molestar y satisfacer en todo al agresor; la segunda fase es la de la agresión propiamente dicha, esta etapa suele durar pocos minutos e incluso algunas horas. Se produce un episodio violento en el que se descarga la tensión acumulada y finaliza cuando el agresor considera que la mujer ha aprendido la lección. La mujer acepta la violencia y espera que termine pronto. La tercera fase se la conoce como reconciliación, en esta fase el hombre se asusta, siente que la mujer lo va a abandonar, así que le dirá o hará cualquier cosa para que le perdone. El maltratador piensa que será capaz de controlarse (Morabes, 2014).

\section{RESULTADO DE INVESTIGACIÓN}

\section{TIPO DE INVESTIGACIÓN:}

Las investigaciones de tipo descriptiva, se considera que analiza e interpreta las funciones o actividades, tomando en cuenta tanto las condiciones como los elementos que caracterizan los procesos, así como las interacciones de las personas que participan en los hogares sujeto de estudio, en este sentido Tamayo y Tamayo (2003). Expresa que las investigaciones descriptivas son aquellas donde no hay manipulación de variables, estas se 
observan y se describen tal como se presentan en su ambiente natural. Su metodología es fundamentalmente descriptiva, aunque puede valerse de algunos elementos cuantitativos y cualitativos. por su parte, es el "cómo" se realizará el estudio para responder al problema planteado, según el tipo el enfoque que se le da a la investigación en lo referente a niveles de explicación que se pueden deducir de sus resultados. En este sentido, las investigaciones se clasifican en exploratorias, descriptivas, correlaciónales, documentales, explicativas, etcétera". (Torres, 2010. p. 290).

\section{MÉTODO DE LA INVESTIGACIÓN}

Según Vergel (1997). El método planteado es el deductivo, lo que implica "El método deductivo consiste en que el investigador practique por medio de conocimientos teóricos sólidos la deducción de verdades preestablecidas para inferir de ellas conclusiones respecto al caso particular.

En este orden de idea, se denotan aspectos lógicos de manera fundamental el objeto inherente a la lógica de la investigación, y por ende al estudio de la metodología a saber: a.- La deducción como una de las formas de inferencia o razonamiento lógico. b.- la aplicación de la lógica formal o la lógica dialéctica, c.- guía el pensamiento del hombre. D.- las conclusiones regidas por diversas reglas generalizadas.

Finalmente, El método de investigación está relacionado con la estrategia general, que guía el proceso de investigación, cuyo, fin es lograr los resultados, específicamente los definidos en los objetivos del estudio. De manera que se puede expresar que el método seleccionado es el deductivo, porque hace parte del enfoque cuantitativo de la investigación.

\section{DISEÑO DE INVESTIGACIÓN}

Hernández, Fernández y Baptista (2001). Exponen que la investigación no experimental es aquella que se realiza sin manipular deliberadamente las variables; es decir, se observan los acontecimientos tal cual como se dan en su contexto natural, para luego ser analizados

Por consiguiente, Hernández et al. (2010), el diseño de la investigación viene a constituir el plan o estrategia que se concibe para alcanzar los objetivos planteados en el estudio, conllevando sus acciones a dar respuestas a los interrogantes presentados en el mismo, así como analizar la certeza de las posturas planteadas en un contexto particular.

Con respecto a lo anterior, Chávez (2001). Indica que las investigaciones de campo se orientan a recolectar información relacionada con el estado actual de las personas, objetos, situaciones o fenómenos, tal cual se presentan al momento de la recolección; es decir, sin manipular o controlar variable alguna, por medio de la técnica de encuesta, la cual se utilizara para recabar la información necesaria para diagnosticar la presencia de la variable sujeta al estudio de investigación planteada en el trabajo investigativo

\section{POBLACIÓN Y MUESTRA}

Según, Arias (2012), define "La población de estudio, como el conjunto finito o infinito de elementos con características comunes para los cuales serán extensivas las conclusiones de la investigación. Esta queda delimitada por el problema y por los objetivos del estudio. Permite conocer desde la población finita, los sujetos a investigar, la se encuentra definida como una agrupación en la que se conoce la cantidad de unidades que la integran. Al mismo tiempo, existe un registro documental de dichas unidades" (p. 81). 


\section{TÉCNICAS E INSTRUMENTOS DE RECOLECCIÓN DE DATOS}

Este método de recolección de datos consiste en el registro sistemático, válido y confiable de comportamientos y situaciones observables, a través de un conjunto de categorías y subcategorías. Útil, por ejemplo, para analizar el aumento de la violencia intrafamiliar, que se presentan en tiempo de pandemia producto del COVID-19, así mismo identificar las manifestaciones que presentan los comportamientos de personas afectadas los cuales de una u otra forma generan inestabilidad en la salud mental al interior del hogar, De lo anterior, En vista de lo anterior se enuncia "Permitirán contar con una narración de los hechos ocurridos (qué, quién, cómo, cuándo y dónde). (Hernández, 2010. p 377)

\section{FUENTES DE RECOLECCIÓN DE LA INFORMACIÓN.}

\section{INSTRUMENTO}

Como instrumento de generación de datos se realizará la entrevista semiestructurada. Es una estrategia mixta que alterna preguntas espontáneas con unas cuantas estructuradas. Este instrumento parte de una guía de preguntas de temas claves que se pretenden investigar. (Martínez, 2011).

\section{FUENTES PRIMARIAS.}

Como fuente primaria se puede determinar la información recolectada en el trabajo de enfoque cuantitativo, mediante la utilización de la técnica, entrevistas e instrumentos como encuesta aplicada a Veinte (20), familias de la comuna de la Ciudad de Riohacha, La Guajira

\section{FUENTES SECUNDARIAS.}

Según las bases teóricas fueron determinantes para la consecución de metas, La información fue tomada de textos.), Páginas Web, monografías referentes al tema, Políticas públicas, metodología de la investigación.

Según Torres (2010), señala "Observación directa Cada día cobra mayor credibilidad y su uso tiende a generalizarse, debido a que permite obtener información directa y confiable, siempre y cuando se haga mediante un procedimiento sistematizado y muy controlado, para lo cual hoy están utilizándose medios audiovisuales muy completos, especialmente en estudios del comportamiento de las personas en sus sitios de trabajo.

Al respeto Hernández (2012), indica un óptimo proceso de recolectar datos implica tres actividades estrechamente vinculadas entre sí: a) Seleccionar un instrumento de medición, el cual debe ser válido y confiable; b) Aplicar ese instrumento de medición; y c) Preparar las mediciones obtenidas para que puedan analizarse correctamente específicamente en las entrevistas, se identifican categorías o grupos de conceptos relevantes para la investigación, con la finalidad de comprender, interpretar, reconstruir y reflexionar acerca de las experiencias e historias de los informantes (Arias, 2012. p. 137)

\section{VALIDEZ Y CONFIABILIDAD DEL INSTRUMENTO}

Según Hernández, Fernández y Batista (2014) la validez designa el grado de medición de un instrumento para determinar la capacidad real de la variable a medir, la cual considera tantos aspectos metodológicos como de contenido. Por ello, para la realización del proceso de validación debe recurrirse a los tipos de validez, donde los de mayor uso están: validez de constructo, de criterio y contenidos. 


\title{
DISCUSIÓN DE RESULTADOS
}

Grafica 1

Entrevista 1 VIF

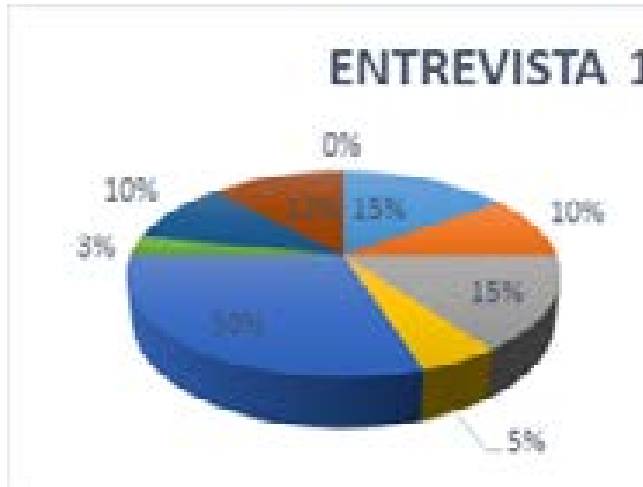

\author{
= CREENCIA DE \\ EFECTACION DEL \\ AGRESOR PARA QUE
}

SUCEDIERA

Fuente. Investigadoras. 2020

La entrevista número uno, presento un $15 \%$ de afectación respecto al relato del suceso de violencia intrafamiliar, un $10 \%$ respecto a las creencias del porque cree que paso la situación, un $15 \%$ referente a los sentimientos de la víctima en el momento de ser violentada o ver el suceso violento, $5 \%$ en las acciones que la entrevistada tomo ante la situación de agresión,
$30 \%$ aumento de violencia intrafamiliar por causa del covid-19, un 3\% referente a las medidas de seguridad que la entrevistada debe tomar en estos casos de VIF, 3 \% Atribución de VIF a situación del victimario $10 \%$ cultura y creencias que hacen prevalente el VIF, y $0 \%$ los conocimientos de la participante respecto a conocimiento sobre centros de ayuda o apoyo de su región.

\section{Grafica 2}

\section{Entrevista 2 VIF}

\section{ENTREVISTA 2 VIF}

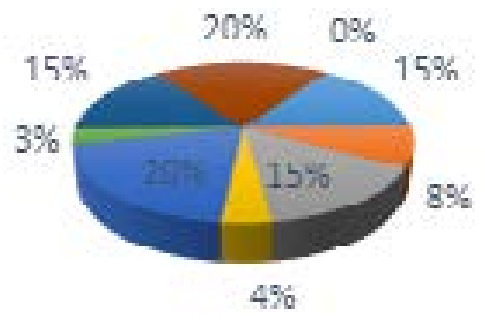

KELAIU SIUACION DE VIOLENCIA

Fuente. Investigadoras. 2020

La entrevista número dos, presento un $15 \%$ de afectación respecto al relato del suceso de violencia intrafamiliar, un $8 \%$ respecto a las creencias del porque cree que paso la situación, un $15 \%$ referente a los sentimientos de la víctima en el momento de ser violentada o ver el suceso violento, $4 \%$ en las acciones que la entrevistada tomo ante la situación de agresión, $20 \%$ aumento de violencia intrafamiliar por causa del covid-19, un $3 \%$ referente a las 
medidas de seguridad que la entrevistada debe tomar en estos casos de VIF, 3 \% Atribución de VIF a situación del victimario $20 \%$ cultura y creencias que hacen prevalente el VIF, y $0 \%$ los conocimientos de la participante respecto a conocimiento sobre centros de ayuda o apoyo de su región.

\section{Grafica 3}

\section{Entrevista 3 VIF}

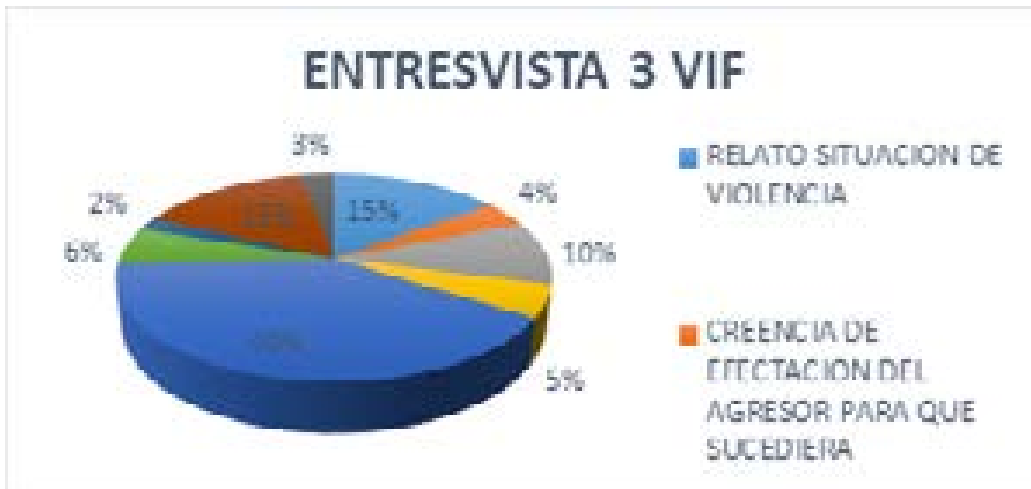

Fuente. Investigadoras. 2020

La entrevista número tres, presento un $15 \%$ de afectación respecto al relato del suceso de violencia intrafamiliar, un $4 \%$ respecto a las creencias del porque cree que paso la situación, un $10 \%$ referente a los sentimientos de la víctima en el momento de ser violentada o ver el suceso violento, $5 \%$ en las acciones que la entrevistada tomo ante la situación de agresión, $40 \%$ aumento de violencia intrafamiliar por causa del covid-19,

\section{Grafica 4}

\section{Entrevista 4 VIF}

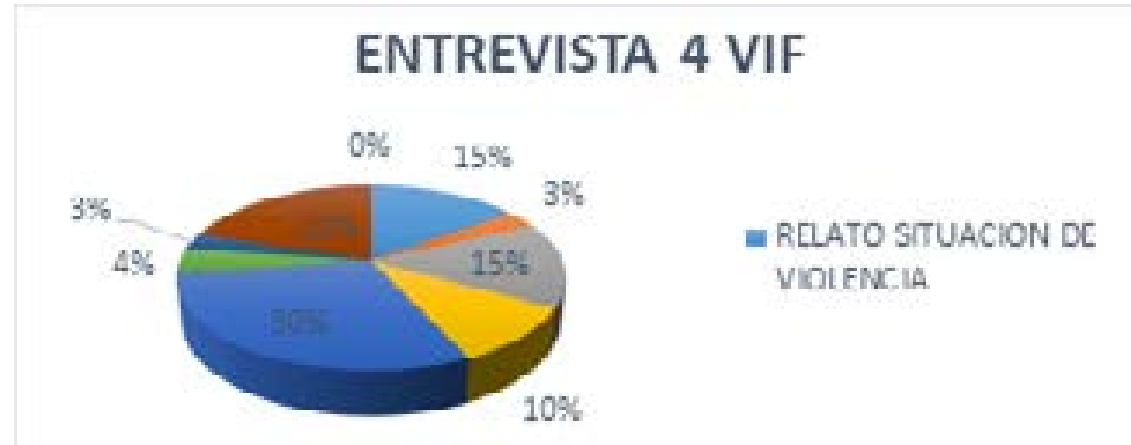

Fuente. Investigadoras. 2020 
La entrevista número cuatro, presento un 15 $\%$ de afectación respecto al relato del suceso de violencia intrafamiliar, un $3 \%$ respecto a las creencias del porque cree que paso la situación, un $15 \%$ referente a los sentimientos de la víctima en el momento de ser violentada o ver el suceso violento, $10 \%$ en las acciones que la entrevistada tomo ante la situación de agresión, 30\% aumento de violencia intrafamiliar por causa del covid-19, un $4 \%$ referente a las medidas de seguridad que la entrevistada debe tomar en estos casos de VIF, 3 \% Atribución de VIF a situación del victimario ,20\% cultura y creencias que hacen prevalente el VIF, y $0 \%$ los conocimientos de la participante respecto a conocimiento sobre centros de ayuda o apoyo de su región.

\title{
Grafica 5
}

Entrevista 5 VIF

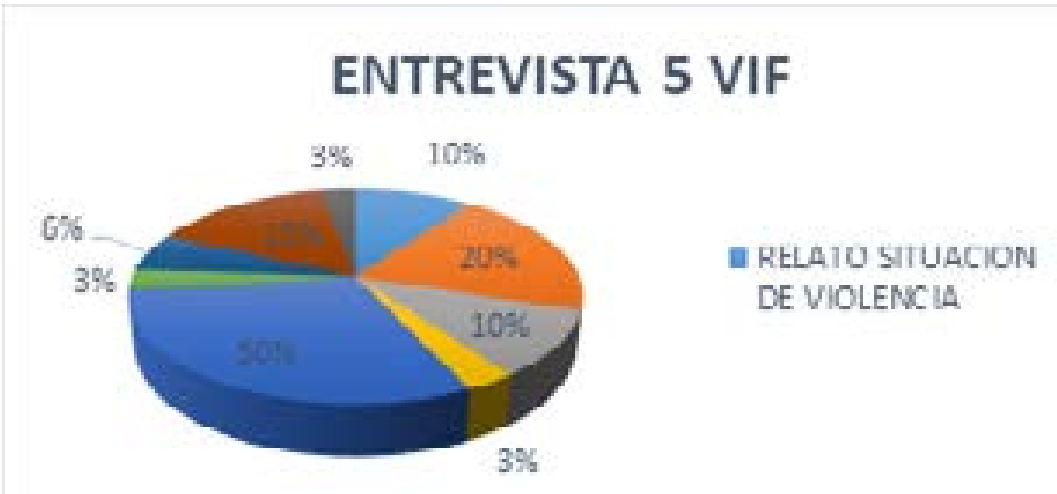

Fuente. Investigadoras. 2020

La entrevista número cinco, presento un $10 \%$ de afectación respecto al relato del suceso de violencia intrafamiliar, un $20 \%$ respecto a las creencias del porque cree que paso la situación, un $10 \%$ referente a los sentimientos de la víctima en el momento de ser violentada o ver el suceso violento, $3 \%$ en las acciones que la entrevistada tomo ante la situación de agresión,
$30 \%$ aumento de violencia intrafamiliar por causa del covid-19, un $3 \%$ referente a las medidas de seguridad que la entrevistada debe tomar en estos casos de VIF, 6 \% Atribución de VIF a situación del victimario, $15 \%$ cultura y creencias que hacen prevalente el VIF, y $3 \%$ los conocimientos de la participante respecto a conocimiento sobre centros de ayuda o apoyo de su región.

\section{Grafica 6}

\section{ENTREVISTA 6 VIF}

\section{ENTREVISTA 6 VIF}

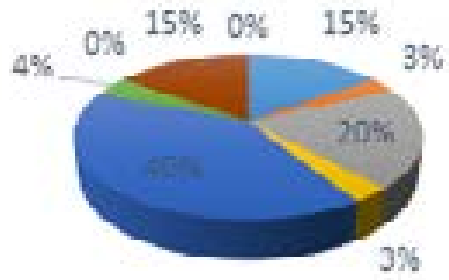

\author{
- RELATO SITUACION \\ DF VIOIFNC. IA
}

Fuente. Investigadoras. 2020 
La entrevista número seis, presento un $15 \%$ de afectación respecto al relato del suceso de violencia intrafamiliar, un $3 \%$ respecto a las creencias del porque cree que paso la situación, un $20 \%$ referente a los sentimientos de la víctima en el momento de ser violentada o ver el suceso violento, $3 \%$ en las acciones que la entrevistada tomo ante la situación de agresión, $40 \%$ aumento de violencia intrafamiliar por causa del covid- 19 , un $4 \%$ referente a las medidas de seguridad que la entrevistada debe tomar en estos casos de VIF, 0 \% Atribución de VIF a situación del victimario, 15\% cultura y creencias que hacen prevalente el VIF, y $0 \%$ los conocimientos de la participante respecto a conocimiento sobre centros de ayuda o apoyo de su región.

\section{ANÁLISIS DE LOS RESULTADOS}

Según El Banco Interamericano de Desarrollo [BID, 2020]. Señala...

"Desde que comenzaran las
medidas de contención del
COVID-19, muchos países han
experimentado un aumento en
los casos de violencia de género.
Las llamadas al teléfono de
emergencia han subido un $80 \%$
en el caso de Colombia, un $43 \%$
en Perú y un $20 \%$ en México. La
situación que hoy vivimos tuvo un
precedente similar en México con
la epidemia de gripe en 2009 .
En aquel entonces escuelas y
oficinas públicas también cerraron
y se constató un crecimiento de la
violencia intrafamiliar"

se destaca los resultados, los cuales son evidentes por el aumento indiscriminado de la violencia intrafamiliar, donde se visibiliza una alarma social, producto de sus características, situaciones conflictivas y problematizadas, que acontecen al interior de la familia, generando alto riesgo en la convivencia familiar y manejo inadecuado de los conflictos, no obstante la incidencia del trastornos, frustraciones, fracasos y el estrés postraumático existente en las diferentes manifestaciones de la violencia intrafamiliar causa ansiedad, temores y depresión, es necesario que la autoridades competentes, diseñen estrategias para asumir decisiones difíciles y dilemas éticos importantes para enfrentar con los equipos interdisciplinarios psicología, trabajo social y otros), para poder establecer prioridades a través de las intervenciones relacionadas con el Covid-19.

\section{LUSIONES}

La situación de contingencia y sus diferentes manifestaciones de violencia intrafamiliar requiere medidas por parte de los diferentes entes gubernamentales, además de la implementación de medidas de salud que propenda mitigar los efectos devastadores en las personas por parte del confinamiento obligatorio, aunque las medidas de protección limiten la intervención o haga que sea más restringido desde el área de la salud mental se pueden implementar herramientas desde las instituciones para abordar los efectos nocivos la violencia intrafamiliar en tiempos de pandemia en el departamento de La Guajira, municipio de Riohacha.

Con relación a la investigación, se precisa que mediante los resultados obtenidos se puede proponer que va direccionada a intervenciones psicosociales, cognitivo conductuales, que intervengan en las respuestas conductuales adecuadas ante situaciones estresoras, aumento del aprendizaje social, terapias cognitivas intervención de las instituciones que han demostrado eficacia en diferentes trastornos y problemas de tipo mental. 


\section{REFERENCIAS Bibliográficas}

Alvarado, A. V. (Septiembre 12, 2018). Cada día se reportan 136 casos de violencia de pareja en Colombia. El Tiempo.com. Obtenido de https://www.eltiempo.com/ justicia/delitos/violencia-de-pareja-encolombia-258066

Arias O. F. (2012). El Proyecto de Investigación Introducción a la metodología científica. $6^{a}$ Edición. ISBN 80-07-8529-9. Editorial Episteme, C.A. Caracas Venezuela

Bernal, C. A. (2010) Metodología de la investigación. Tercera edición. Pearson Educación. ISBN: 978-958-699-128-5. Área: Metodología. Colombia

Comisión Económica para América Latina y el Caribe (CEPAL,2016) Violencia Física, psicológica contra la mujer en relación de pareja. Obtenido de Violencia basada en Género: https://oig.cepal.org/sites/ default/files/mimp_violencia_basada_ en_genero_marco_conceptual_para_ las_politicas_publicas_y_la_accion_ del_estado.pdf

Corsi J, y Bobino L. 2014. Violencia y género: la construcción de la masculinidad como factor de riesgo. En: Violencias Sociales. Estudios sobre Violencia. Barcelona: Editorial Ariel

Departamento de Comunicación Global de las Naciones Unidas. (DCGNU, 2020). Víctimas de la violencia doméstica atrapadas durante la cuarentena. Obtenido de https://www.un.org/es/ coronavirus/articles/un-supportingtrappeddomesticviolence-victimsduring-covid-19-pandemic

Flores, M. C., y González, A. A. (2015). Caracterización de la violencia intrafamiliar, maltrato infantil y abuso sexual en Bogotá DC durante el año 2011. Teoría y Praxis Investigativa, 8(1), 74-91

Gallego, A. (2011). Recuperación crítica de los conceptos de familia, dinámica familiar y sus características. Revista Virtual Universidad Católica del Norte, (35), 326-345.

Gómez Macfarland. Carla Angélica \& Sánchez Ramírez. María Cristina 2020. Violencia familiar en tiempos de Covid. Revista. Mirada Legislativa. No 187. Instituto Belisario Domínguez. Dirección Nacional de Análisis Legislativo. Senado de la República. México

Hernández Sampieri. Roberto. Metodología de la Investigación. Quinta edición derechos reservados @ 2010, 2006, 2003, 1998, 1991 respecto a la quinta edición por: McGraw

Hernández et al, (2014) El Maltrato Psicológico. Causas, Consecuencias y Criterios Jurisprudenciales. El Problema Probatorio, Universidad de alicante.

Ministerio de Salud y Protección Social (MinSalud, 2020) p. 5-8

Organización Mundial de la Salud (OMS, 2012). Informe mundial sobre la violencia y la salud. Washington, D.C.: s.n.

Palacio, M. (2004). Fundación Fesco. Recuperado el 5 de agosto de 2020 de https://bit.ly/3kM1Lif

Pavón, S. Santamaría, M. (2010) Patrones relacionados de violencia intrafamiliar. Lectura sistemática de una experiencia. Quito-Ecuador, ABYAYALA Editorial.

Prins, B. (0ctubre 31, 2017). Sigue en aumento la violencia de género en el Departamento: 844 casos en lo que va del 2017. Diario 
del Norte. Obtenido de https://www. diariodelnorte.net/noticias/la-guajiranoticias/sigue-en-aumento-la-violenciade-genero-en-el-departamento-844casos-en-lo-que-va-del-2017/

Ruiz Varea, J. 2016. Crimipedia: Violencia Intrafamiliar. Obtenido de http:// crimina.es/crimipedia/wpcontent/ uploads/2016/10/Violenciaintrafamiliar.Juan-Ruiz-Varea.pdf

S. Morabes. 2014. Ciclo de violencia en la asistencia psicológica a víctimas de violencia de género. Políticas públicas e inclusión en las democracias contemporáneas, Volumen (1), pp. 1

J. Whaley. 2003. Violencia intrafamiliar. Causas biológicas, psicológicas, e comunicacionales e interaccionales. México D.F. - México. 